\title{
A Fitting Algorithm for Random Modeling the PLC Channel
}

\author{
Andrea M. Tonello. \\ Benjamín Béjar
}

\author{
Fabio Versolatto \\ Santiago Zazo
}

\begin{abstract}
The characteristics of the power-line communication (PLC) channel are difficult to model due to the heterogeneity of the networks and the lack of common wiring practices. To obtain the full variability of the PLC channel, random channel generators are of great importance for the design and testing of communication algorithms. In this respect, we propose a random channel generator that is based on the top-down approach. Basically, we describe the multipath propagation and the coupling effects with an analytical model. We introduce the variability into a restricted set of parameters and, finally, we fit the model to a set of measured channels. The proposed model enables a closed-form description of both the mean path-loss profile and the statistical correlation function of the channel frequency response. As an example of application, we apply the procedure to a set of in-home measured channels in the band 2-100 MHz whose statistics are available in the literature. The measured channels are divided into nine classes according to their channel capacity. We provide the parameters for the random generation of channels for all nine classes, and we show that the results are consistent with the experimental ones. Finally, we merge the classes to capture the entire heterogeneity of in-home PLC channels. In detail, we introduce the class occurrence probability, and we present a random channel generator that targets the ensemble of all nine classes. The statistics of the composite set of channels are also studied, and they are compared to the results of experimental measurement campaigns in the literature.
\end{abstract}

Index Terms-Channel modeling, power line communications, random channel generation, top-down approach.

\section{INTRODUCTION}

$\mathbf{N}$ O NEW wires is the essence of power-line communication (PLC). PLC conveys information through the existing power delivery infrastructure and it is a very attractive solution for providing a connection to the end user. Several application scenarios can be found. Among these, the mediumvoltage (MV), the outdoor low-voltage (LV), and the in-home scenarios have been the subject of high research activity during the last decade. In this paper, we focus on the in-home broadband scenario.
Inhome PLC is a candidate to overcome the range limitations of wireless networks, ensuring communications at $200 \mathrm{Mb} / \mathrm{s}$ in the 2-30 MHz frequency band with available commercial devices [1]. To further increase the data rate, the signaling bandwidth can be extended to $2-100 \mathrm{MHz}$, as it is done, for instance, in the next-generation standard G.hn [2]. In this respect, a broadband channel model in the extended frequency range is important.

A large effort has been dedicated to model the power-line channel, but no reference model has been provided yet. There are two main approaches. The first is referred to as bottom up. Basically, the bottom-up approach models the channel transfer function using transmission-line (TL) theory. It requires perfect knowledge of the network topology in terms of cable lengths, loads, and so on. In general, the network elements are described with the $\mathrm{ABCD}$ or the scattering parameter matrices [3], [4]. Alternative solutions were presented in [5] and [6]. The first solves a complex system and it finds the current and voltage of every node of the network. The latter is a scalar version of the ABCD matrix method.

The bottom-up approach admits a statistical extension. Random channel generation algorithms were presented in [7] and [8] for the frequency range up to $30 \mathrm{MHz}$. Furthermore, the bottom-up approach allows modeling the time-variant nature of the PLC channel. The random generation of time-variant PLC channels was addressed in [9]. Finally, the bottom-up approach can be extended to multiple-input multiple-output (MIMO) PLC. In fact, in-home PLC deploys three conductors. With three conductors, two circuits are available and, thus, MIMO communications are possible. MIMO PLC models were described in [10] and [11], and they represent the natural extension of [5] and [6], respectively.

The second modeling approach is referred to as top down. With this approach, the channel response is obtained by fitting a certain parametric function with data coming from the measurements. The first attempt was presented in [12]. Later, in [13], the channel frequency response was modeled taking into account the multipath nature of the signal propagation and the losses of the cables. In [14], the model was extended in statistical terms and a top-down random channel generator was provided. Some other top-down random channel generation algorithms were presented in the literature. In [15], a statistical model for the PLC channel impulse response was derived from the statistics of the delay spread and the attenuation of the set of measured channels that was presented in [16]. Conversely, the channel generation in the frequency domain was addressed in [17]. Basically, the method generates channel responses that 
show the same distribution of peaks and notches of the measured channels. The work targets the extended frequency range up to $100 \mathrm{MHz}$. The main disadvantage of the method is that the position and the height of the peaks and notches is strongly modified by the interpolation in frequency of the channel transfer function, as was pointed out in [18].

In this paper, we refine the model presented in [14], and we propose a general fitting procedure that enables the model to reproduce the statistics of a given set of measured channels. We target channel frequency responses in the $2-100 \mathrm{MHz}$ frequency band. We show that the model enables obtaining the analytical expression of the mean path-loss profile and the statistical correlation function of the generated channels. These are important functions that we propose to exploit in the fitting process.

As an application example of the proposed method, we consider the results of the measurement campaign in [17] where the measured channels were classified into nine classes according to their capacity. We fit the model to the measures, we study the statistics of the generated channels, and we show that it is in good agreement with the experimental one. In [19], some preliminary results were reported for a restricted subset of channel classes. Herein, we extend the validity of these results. We derive the closed-form expression of the statistical correlation function of the channel frequency response. From it, we obtain the mean path-loss profile and the coherence band. We detail the fitting procedure, and we provide the parameters of the model for all channel classes that were presented in [17]. We study the statistics of the generated channels and, in particular, the distribution of the delay spread and the average channel gain. As a final result, we show their consistency with experimental results. Furthermore, we propose using a composition channel where the frequency-response realizations are drawn from the nine classes with the class occurrence probabilities that were reported in [17]. This enables capturing the full in-home PLC channel variability.

The remainder of this paper is organized as follows. In Section II, we deal with the model. First, we recall the multipath propagation model. Then, we extend it in statistical terms. In Section III, we describe the fitting procedure and the application to the results of the measurement campaign in [17]. In Section IV, we provide the statistical analysis of the generated channels and we compare it with experimental results. Finally, some conclusions follow.

\section{MODEL DESCRIPTION}

We model the channel response in the frequency domain. First, we recall the basis of the multipath propagation model. Then, we propose a statistical extension that enables generating random PLC channel responses, whose statistics are in agreement with experimental results.

\section{A. Deterministic Propagation Model}

We aim to describe the multipath propagation of the signal in power-line networks. We focus on the in-home scenario. The in-home wiring is made by distinct conductors. Two of these, namely, the phase and the neutral, deliver the electricity and they are also deployed for communication purposes. The bare conductors are individually insulated and they can be either enclosed in the same dielectric cap or placed inside small plastic raceways. In both cases, the transversal dimension of the overall cable structure is small if compared to the transmission signal wavelength in the lower gigahertz range. Furthermore, the dielectric can be approximated as uniform, and the wires as ideal conductors. It follows that the transverse electromagnetic (TEM) or quasi-TEM mode assumption is valid and, thus, the electrical quantities along the line can be handled as scalars.

Inhome power delivery networks are characterized by the presence of a multitude of branches and termination outlets. The electrical appliances are connected to the termination outlets. In general, they show an input impedance that is different from the characteristic impedance of the cables and, thus, they are unmatched terminations. Note that open outlets (i.e., plugs where no loads are connected) are unmatched terminations as well. Cable junctions and unmatched terminations can be modelled as line discontinuities. On a line discontinuity, the signal is partially reflected toward the transmitter and it is partially transmitted over the discontinuity. The reflection and transmission coefficients account for these effects. We denote them with $\rho(f)$ and $\tau(f)$, respectively, where $f$ is the frequency.

In the presence of multiple line discontinuities, infinite copies of the transmitted signal propagate toward the receiver. Each copy follows a different path with its own reflection effects. We denote the phasor vector of the signal at the transmitter and the receiver port with $V_{t x}(f)$ and $V_{r x}(f)$, respectively. The signal at the receiver port can be written as [20]

$$
V_{r x}(f)=\sum_{i=1}^{N} \underbrace{\left(\prod_{m=1}^{R_{i}} \rho_{m}(f) \prod_{n=1}^{T_{i}} \tau_{n}(f)\right)}_{p_{i}(f)} e^{-\gamma(f) \ell_{i}} V_{t x}(f)
$$

where $N$ is the number of paths, while $R_{i}, T_{i}$, and $\ell_{i}$ are the number of reflection and transmission coefficients and the length of the $i$ th path, respectively. The exponential factor accounts for the propagation effects. We denote with $\gamma(f)$ the propagation constant. It is, in general, complex, namely $\gamma(f)=\alpha(f)+j \beta(f)$, and it is a function of the cable characteristics. We assume all lines have the same propagation constant. The real component (i.e., $\alpha(f)$ ) is the attenuation constant and it accounts for the losses introduced by the nonideal lines. The imaginary component $\beta(f)$ is the phase constant. We model the attenuation and the phase constant as [13]

$$
\begin{aligned}
& \alpha(f)=a_{0}+a_{1} f^{K} \\
& \beta(f)=\frac{2 \pi f}{\nu}
\end{aligned}
$$

where $a_{0}, a_{1}$, and $K$ are a function of the cable characteristics, $\nu=c / \varepsilon_{r}$ is the propagation speed of light in the cable structure, $c$ is the speed of light in the vacuum, and $\varepsilon_{r}$ is the relative dielectric constant of the insulator that surrounds the conductors. In the following text, we choose a value for the relative dielectric constant that takes the nonuniform dielectric into account, given by the combination of air and plastic. Strictly, we assume $\varepsilon_{\text {r }}=1.5$.

We refer to the product of the reflection and the transmission coefficients as path gain. We denote the path gain of the $i$ th path with $p_{i}(f)$. In general, the path gains are complex and smaller 


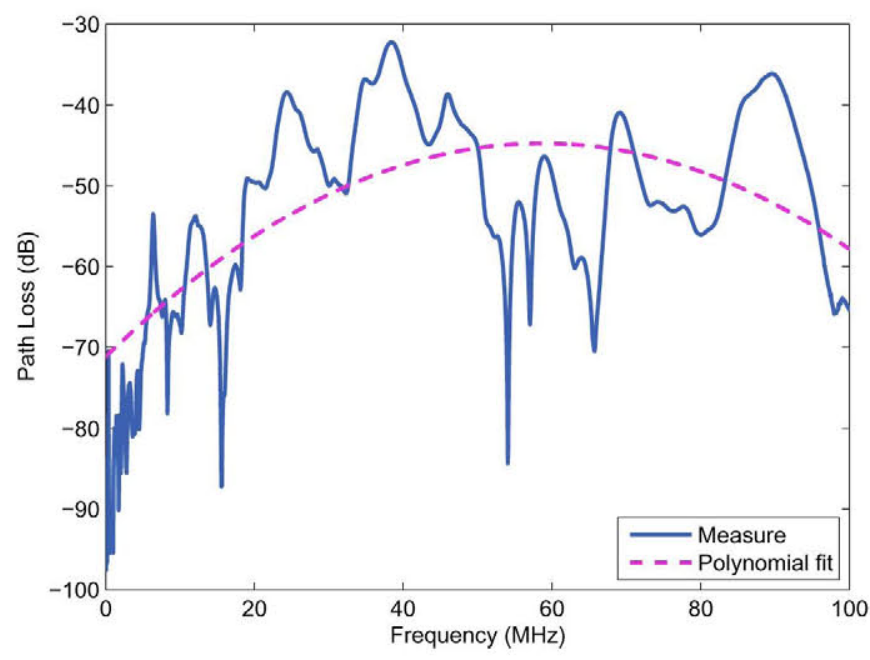

Fig. 1. Measure and quadratic polynomial fit of a PLC channel frequency response that shows concave behavior. The measurement was made in the university laboratories.

than one in absolute value. However, for the cases of practical interest, $p_{i}(f)$ can be approximated as a real-valued coefficient [13]. Further, we propose making the frequency dependence of the path gains explicit as follows:

$$
p_{i}(f)=g_{i}+c_{i} f^{K_{2}}
$$

where $K_{2}$ is the same for all paths. The model in (4) has been first presented in [21], and it can be explained as follows. Inhome networks are fed by one or more phases, namely, up to three. The phases are not electrically connected. When more than one phase is present, the outlets do not necessarily share the same phase. Thus, they are connected to different electrical circuits. When we transmit and receive on different circuits, coupling effects ensure the propagation of the transmitted signal toward the receiver. In the presence of coupling effects, the channel frequency response shows a concave behavior. In detail, it is strongly attenuated in the lower frequency range because it lacks electrical continuity, while in the higher frequency range, it is attenuated because of line losses. From the measurements, we noted that concave frequency responses can also be found in single-phase networks where the transmitter or the receiver outlet are partially damaged. As an example, in Fig. 1, we show a measured frequency response that exhibits typical concave behavior, and its best quadratic polynomial fit.

We neglect the paths that are characterized by a small path gain and, thus, we limit (1) to the finite number of paths $N_{p}$. Now, the complex channel frequency response is given by the ratio between the phasor of the received signal and the phasor of the transmitted signal (i.e., $H(f)=V_{r x}(f) / V_{t x}(f)$ ). From (1), it follows:

$$
H(f)=A \sum_{i=1}^{N_{p}}\left(g_{i}+c_{i} f^{K_{2}}\right) e^{-\left(a_{0}+a_{1} f^{K}\right) \ell_{i}} e^{-j 2 \pi f \ell_{i} / \nu}
$$

where $A$ is a constant coefficient that enables attenuation adjustments, and $0 \leq B_{1} \leq f \leq B_{2}$. In the following text, we consider the frequency response in (5) and, thus, we no longer use the phasor representation.
Now, we focus on the channel impulse response. We define the complex impulse response of the channel as the inverse Fourier transform of the frequency response in (5) (i.e., $h(t)=$ $\left.\mathcal{F}^{-1}[H(f)]\right)$. Under the assumption of $c_{i}=0$ and $K=1$, it is possible to obtain a closed-form expression of the complex channel impulse response, that reads

$$
h(t)=A \sum_{i=1}^{N_{p}} \sum_{k=1}^{2} g_{i} e^{-a_{0} \ell_{i}} \frac{(-1)^{k-1} e^{\left(j t-\left(a_{1} / 2 \pi+j / \nu\right) \ell_{i}\right) \omega_{k}}}{a_{1} \ell_{i}+j 2 \pi\left(\frac{\ell_{i}}{\nu}-t\right)}
$$

where $\omega_{k}=2 \pi B_{k}$. When the assumptions are not valid, the complex impulse response of the channel can be computed as the inverse discrete Fourier transform (IDFT) of the channel frequency response.

Finally, the real impulse response of the channel is given by twice the real part of the complex impulse response, i.e., $h_{r}(t)=$ $2 \operatorname{Re}\{h(t)\}$.

\section{B. Proposed Statistical Extension of the Model}

In this paper, we focus on the in-home scenario, and we propose generating random channel responses starting from (5). We fix the value of the parameters $A, a_{0}, a_{1}, K, K_{2}$, and $\nu$, and we model $g_{i}, c_{i}, \ell_{i}$, and $N_{p}$ as random variables. We obtain the values of the constants by fitting the measures as explained in Section III, and we choose the distribution of the random variables as follows.

First, we focus on the path gain coefficients $g_{i}$ and $c_{i}$. The path gains are given by the product of the reflection and transmission coefficients. We assume the latter to be uniformly distributed random variables in $[-1,1]$. Therefore, we can model them as the product of a random sign flip $\xi$ and a uniformly distributed random variable $u \in[0,1]$. We note that the null reflection and transmission coefficients do not contribute to the sum in (5). Now, since the statistical distribution of the product of a large number of uniform random variables approaches log-normality, we model $g_{i}$ and $c_{i}$ as log-normally distributed random variables multiplied by random sign flips. Differently, in [21], $g_{i}$ and $c_{i}$ were modeled as uniformly distributed random variables in $[-1,1]$. We assume $c_{i}$ and $g_{i}$ to be independent, and we point out that $g_{i}$ and $c_{i}$ have zero mean. We denote their variances with $\sigma_{g}^{2}$ and $\sigma_{c}^{2}=b_{0}^{2} \sigma_{g}^{2}$, respectively. Finally, without any loss of generality, we set $\sigma_{g}^{2}=1$. Further attenuation adjustments can be obtained by properly setting the value of the constant coefficient $A$.

We model the path lengths as uniformly distributed random variables in $[0, L]$, where $L$ is constant. Finally, we propose modeling the number of paths $N_{p}$ as a Poisson random variable with mean $\Lambda L$. The number of paths in (5) is always greater than 0 . Therefore, the probability mass function of $N_{p}$ reads

$$
\operatorname{Pr}\left(N_{p}=k\right)=\frac{e^{-\Lambda L}}{1-e^{-\Lambda L}} \frac{(\Lambda L)^{k}}{k !}
$$

where $k \geq 1$. The paths can be interpreted as the arrivals of a Poisson process with intensity $\Lambda$ (paths $/ \mathrm{m}$ ). In fact, the arrivals of a Poisson process are uniformly distributed in a finite interval, when their number is set. In the following text, we assume $\Lambda=$ 0.2 . 
This model is able to reproduce the reality with accurate precision, as will be shown in Section IV. However, we remark that other distributions may be suitable for $N_{p}$, depending on the set of measures that have to be fitted.

\section{Fitting the Model to the Measurements}

We herein describe a procedure to obtain the model parameters that fit the measured channels. The idea is to target the average path loss and the coherence bandwidth of a set of measured channels. We compute the coherence bandwidth from the statistical frequency correlation function that will be described. As an application example, in this paper, we fit the model to the results of the measurement campaign in [17].

\section{A. Statistical Frequency Correlation Function and Path Loss}

We define the statistical frequency correlation function as follows:

$$
\phi(f, \lambda)=E\left[H(f+\lambda) H^{*}(f)\right]
$$

where $E[\cdot]$ denotes the expectation in regards to the random variables, and the superscript $\{\cdot\}^{*}$ denotes the complex conjugate. From (5), we obtain (see Appendix)

$$
\begin{aligned}
\phi(f, \lambda)=(1+ & \left.b_{0}^{2} f^{K_{2}}(f+\lambda)^{K_{2}}\right) \frac{|A|^{2} \Lambda}{1-e^{-\Lambda L}} \\
& \times \frac{1-e^{-\left(2 a_{0}+a_{1}\left(f^{K}+(f+\lambda)^{K}\right)+j 2 \pi \lambda / \nu\right) L}}{2 a_{0}+a_{1}\left(f K+(f+\lambda)^{K}\right)+\frac{j 2 \pi \lambda}{\nu}} .
\end{aligned}
$$

When $\lambda=0,(9)$ corresponds to the mean path-loss profile of the generated channels, namely, $P(f)=\phi(f, 0)$, that reads

$$
P(f)=\frac{|A|^{2} \Lambda\left(1+b_{0}^{2} f^{2 K_{2}}\right)\left(1-e^{-2\left(a_{0}+a_{1} f^{K}\right) L}\right)}{2\left(1-e^{-\Lambda L}\right)\left(a_{0}+a_{1} f^{K}\right)} .
$$

\section{B. Fitting Procedure}

We aim to fit the model in (5) to a set of measured channels. We proceed as follows. We find the values of the parameters and the distributions of the random variables in (5) that minimize the mean square error between the average path-loss profile of the measured channels and the analytical profile in (10). We constrain the minimization to target the statistical coherence bandwidth of the measured channels. We define the statistical coherence bandwidth as follows. First, we integrate the statistical correlation function in (9) to obtain

$$
\bar{\phi}(\lambda)=\int_{B_{1}}^{B_{2}} \phi(f, \lambda) d f
$$

Then, we refer to the statistical coherence bandwidth at level $\varphi$, namely, $\hat{B}_{C}^{(\varphi)}$, as the frequency $\lambda_{\varphi}$ beyond which the absolute value of $\bar{\phi}$ falls to a value that is $\varphi$ times its maximum. Strictly

$$
\hat{B}_{C}^{(\varphi)}=\lambda_{\varphi} \quad \text { such that } \quad\left|\bar{\phi}\left(\lambda_{\varphi}\right)\right|=\varphi|\bar{\phi}(0)|
$$

TABLE I

COUPLING PARAMETER VALUeS

\begin{tabular}{ccc}
\hline class & $b_{0}^{2}$ & $K_{2}$ \\
\hline 1 & $1.4354 \mathrm{e}-6$ & 0.403919 \\
$2-8$ & 0 & - \\
9 & $2.28955 \mathrm{e}-6$ & 0.341468 \\
\hline
\end{tabular}

Now, the statistical coherence bandwidth is not always reported in experimental works. Typically, the main focus is on the coherence bandwidth $B_{C}^{(\varphi)}$ that is defined similar to (12), but substituting $\bar{\phi}(\lambda)$ with the following correlation function:

$$
R(\lambda)=\int_{B_{1}}^{B_{2}} H(f+\lambda) H^{*}(f) d f
$$

where $H(f)=0$ outside the frequency range $\left[B_{1}, B_{2}\right]$. Note that $R(\lambda)$ and $\bar{\phi}(\lambda)$ are different. The former refers to a specific channel $H(f)$, the latter is the result of expectation.

When the statistical coherence bandwidth of the measured channels is not available, we propose fitting the model so that the statistical coherence bandwidth has a value equal to the average value of the coherence bandwidth of the measured channels that we denote with $\bar{B}_{C}^{(\varphi)}$. The proposed approach is valid because $\bar{B}_{C}^{(\varphi)}$ and the statistical coherence bandwidth $\hat{B}_{C}^{(\varphi)}$ of the generated channels are very close quantities, as will be shown in Section IV.

\section{Target Measurement Campaign}

In the literature, several works report the results of different measurement campaigns [18], [22]. Most of these focus on the characterization of the PLC channel in terms of average channel gain and delay spread. To fit the model, we need the average path-loss profile and the average coherence bandwidth. In [17] and [23], this information is provided. Therefore, we target the database herein presented. Basically, the work in [17] and [23] addresses the in-home PLC scenario in France. The campaign focuses on the $2-100 \mathrm{MHz}$ frequency band. A set of 144 channels was acquired in different locations, and the channels were classified into nine channel classes according to their capacity. This classification is useful because it allows inferring the statistics of groups of channels that show similar frequency behavior. For each class, the average path-loss profile, delay spread, and coherence bandwidth are given. Furthermore, a hyperbolic relation between the average delay spread and coherence bandwidth is provided.

\section{NUMERICAL RESULTS}

We fit the model to the nine-channel classes in [17]. We focus on the $2-100 \mathrm{MHz}$ frequency range, with a frequency resolution of $24 \mathrm{kHz}$. In the time domain, we sample the real impulse response with a period of $T_{c}=5 \mathrm{~ns}$. We report the parameter values in Tables I and II. Furthermore, following the fitting procedure of Section III-B, we refine the values provided in [19] for classes 1,5 , and 9 . 
TABLE II

ATtenuation and Multipath Parameter Values $\left(\sigma_{g}^{2}=1, \quad \Lambda=0.2\right.$ paths $\left./ \mathrm{m}, \quad \nu=2 e 8 \mathrm{~m} / \mathrm{s}\right)$

\begin{tabular}{cccccc}
\hline class & $A$ & $\begin{array}{c}a_{0} \\
\left(m^{-1}\right)\end{array}$ & $\begin{array}{c}a_{1} \\
\left(s \cdot m^{-1}\right)\end{array}$ & $K$ & $\begin{array}{c}L \\
(m)\end{array}$ \\
\hline 1 & $1.3022 \mathrm{e}-5$ & -0.00691505 & $1.15712 \mathrm{e}-026$ & 2.97983 & 540 \\
2 & $2.8269 \mathrm{e}-4$ & -0.00888846 & $7.55014 \mathrm{e}-006$ & 0.408174 & 550 \\
3 & $6.7170 \mathrm{e}-4$ & -0.0152108 & $3.67885 \mathrm{e}-005$ & 0.347786 & 320 \\
4 & $6.3972 \mathrm{e}-4$ & -0.0142857 & $2.5219 \mathrm{e}-005$ & 0.348188 & 350 \\
5 & $8.3880 \mathrm{e}-4$ & -0.0141565 & $1.67181 \mathrm{e}-005$ & 0.363295 & 350 \\
6 & $9.5814 \mathrm{e}-4$ & -0.00797313 & $2.285 \mathrm{e}-018$ & 1.92048 & 410 \\
7 & $4.5819 \mathrm{e}-3$ & -0.0132538 & $1.12949 \mathrm{e}-018$ & 2.00313 & 200 \\
8 & $1.0964 \mathrm{e}-2$ & -0.0185199 & $9.65172 \mathrm{e}-018$ & 1.87202 & 130 \\
9 & $2.4856 \mathrm{e}-3$ & -0.0435673 & $2.02324 \mathrm{e}-020$ & 2.2179 & 110 \\
\hline
\end{tabular}

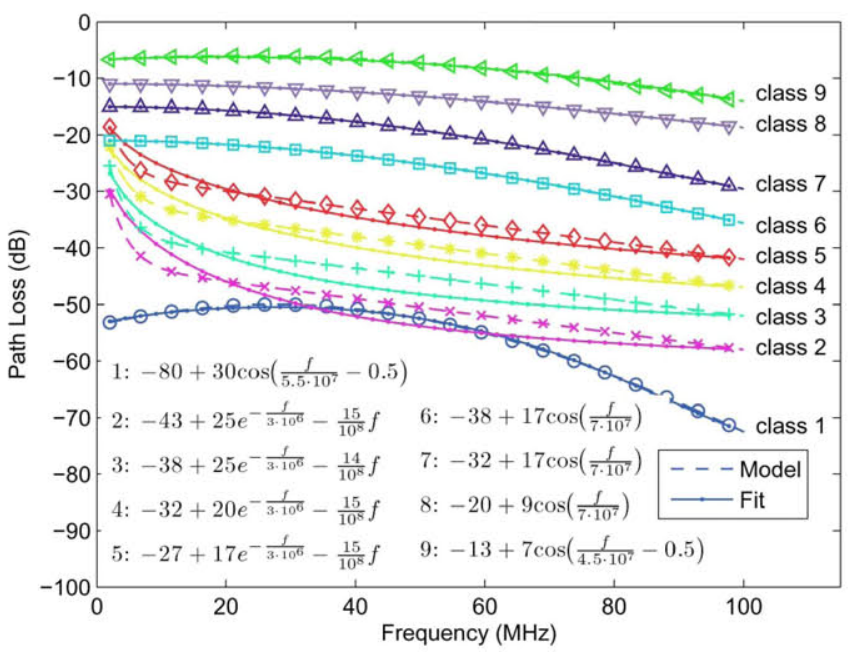

Fig. 2. Target path-loss profiles (dashed line with markers and explicit formulas) and profiles from fitting (continuous line) for channel classes 1 to 9 .

\section{A. Path Loss and Phase}

We first study the path loss of the generated channels. For each channel class, 500 realizations are considered. In Fig. 2, we plot the mean path-loss profile obtained from the measurements [17], and the analytical path loss given by (10). The closed-form expression of the measured average path loss is provided in [17] and it is also reported in Fig. 2. It has been obtained by fitting with sinusoidal or exponential functions the average path loss of the measured channels. In all cases, a very good agreement between the analytical (of our model) and the measured path loss can be observed. It validates the results of the fitting procedure.

Now, we consider the average phase. We compute it as the average phase of the channel frequency response of the generated channels. We report the average phase of the nine classes in Fig. 3. In most cases, the average phase is well fitted by a linear function, as reported in [17]. Only classes 2 and 3 show a slight quadratic dependency of the phase versus frequency.

Finally, in Fig. 4, we show an example of channel realization from classes 2 and 8 , both in amplitude and phase. The average profiles are also shown.

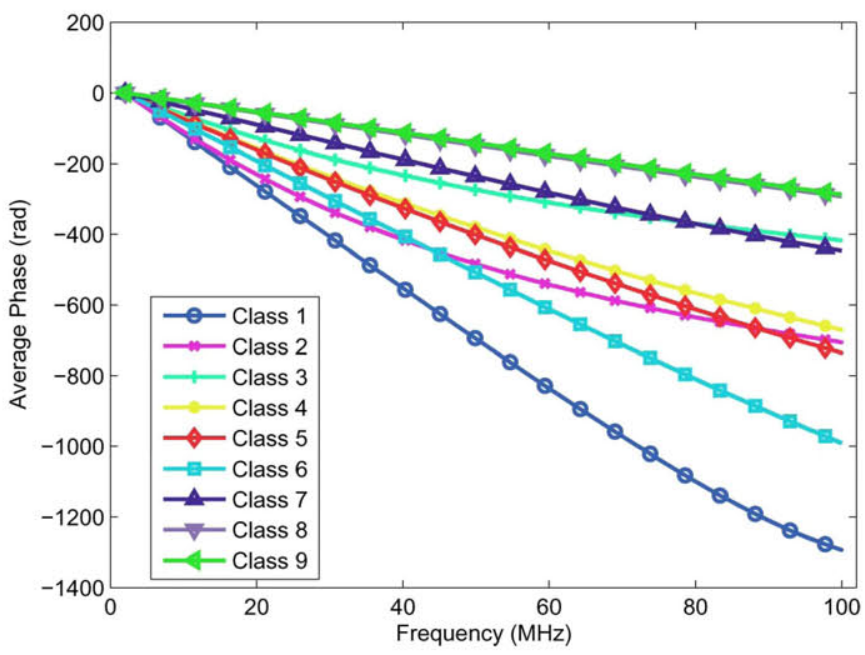

Fig. 3. Average phase of classes 1 (bottom) to 9 (top).

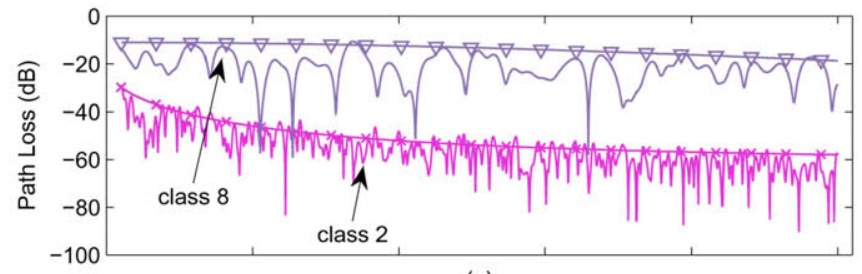

(a)

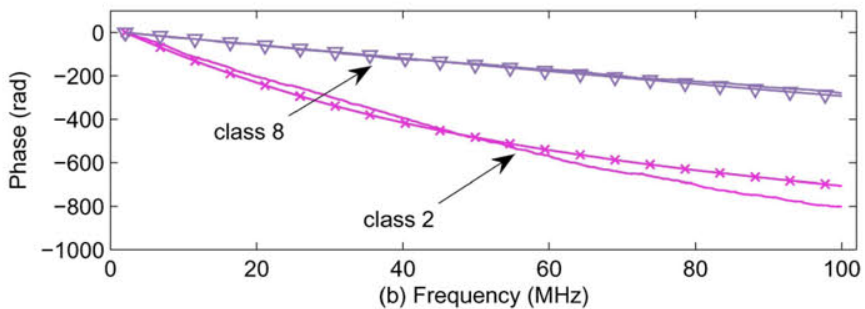

Fig. 4. Illustrative example of one channel realization from classes 2 and 8 . (a) Amplitude. (b) Phase. The average profile in amplitude and phase is also shown for both classes (lines with markers).

\section{B. Statistics of the Channel Metrics}

We first focus on the root-mean-square (rms) delay spread. We compute the power delay profile from the real impulse response as

$$
\mathcal{P}(t)=\frac{\left|h_{r}(t)\right|^{2}}{\int_{0}^{\infty} \tau\left|h_{r}(\tau)\right|^{2} d \tau} .
$$

Then, the delay spread reads

$$
\sigma_{\tau}=\sqrt{\int_{0}^{\infty}\left(t-m_{\tau}\right)^{2} \mathcal{P}(t) d t} \quad[\mathrm{~s}]
$$

where $m_{\tau}=\int_{0}^{\infty} t \mathcal{P}(t) d t$ is the mean delay. We compute the channel impulse response by means of IDFT, and we truncate it to $5.56 \mu \mathrm{s}$ to reduce the side-lobe effect. The truncation introduces negligible energy loss. Furthermore, it is consistent with 


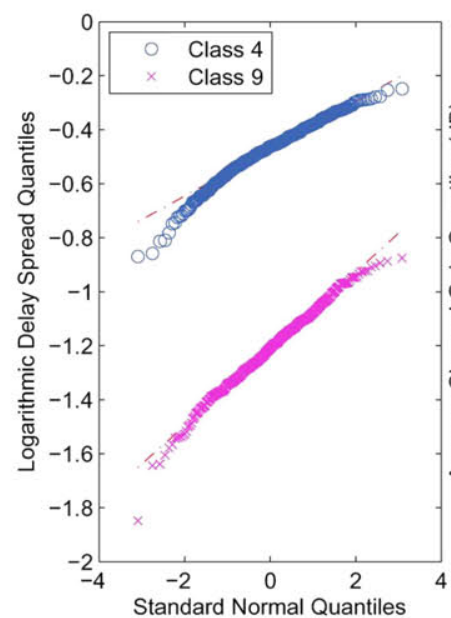

(a)

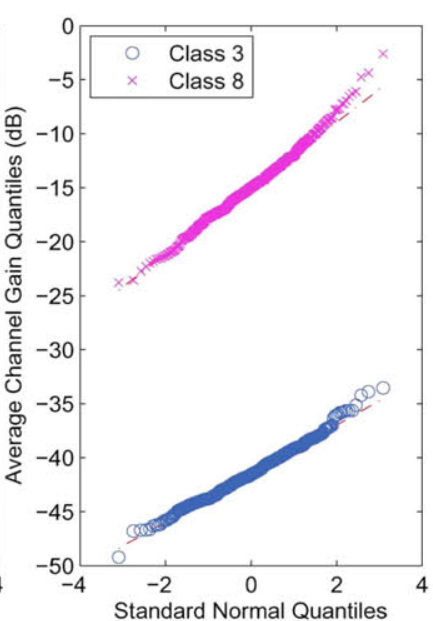

(b)
Fig. 5. (a) Quantile-quantile plot of the logarithmic delay spread versus the standard normal quantiles. (b) Quantile-quantile plot of the average channel gain versus the standard normal quantiles. In both cases, results are provided for two representative classes.

TABLE III

RMS DELAY SPREAD $(\mu \mathrm{s})$ AND ACG $(\mathrm{dB})$

\begin{tabular}{cccc}
\hline class & Measured [23] & \multicolumn{2}{c}{ Simulated } \\
& $\bar{\sigma}_{\tau}$ & $\bar{\sigma}_{\tau}$ & $\bar{G}$ \\
\hline 1 & 0.31 & 0.399061 & -53.8721 \\
2 & 0.517 & 0.601574 & -44.8233 \\
3 & 0.456 & 0.384516 & -41.4305 \\
4 & 0.297 & 0.344387 & -36.0103 \\
5 & 0.322 & 0.321122 & -31.4896 \\
6 & 0.263 & 0.33135 & -25.2677 \\
7 & 0.149 & 0.190027 & -19.4345 \\
8 & 0.0999 & 0.127396 & -14.8919 \\
9 & 0.0417 & 0.0643916 & -10.0729 \\
\hline
\end{tabular}

the cyclic-prefix length of HomePlug AV PLC system specifications [1]. A raised cosine window with a flat portion in the $2-100 \mathrm{MHz}$ frequency band is applied.

In Table III, we provide the average delay spread of the nine channel classes. We denote it with $\bar{\sigma}_{\tau}$. We compare the values obtained from the simulations (third column) to the ones given by the measures (second column). The measured values were presented in [23], and we report them here for the sake of clarity. Simulations are very close to the measures. Furthermore, we study the distribution of the delay spread of the generated channels. We perform the Lilliefors test to the logarithm version of the delay spread (i.e., $\sigma_{\log }=\log _{10}\left(\sigma_{\tau} / 1 \mu \mathrm{s}\right)$ ). The delay spread of class 1,5 , and 9 is $\log$-normally distributed. For other classes, the Lilliefors test does not confirm the log-normality of $\sigma_{\tau}$, though the log-normal distribution is still the best fit. In Fig. 5(a), we show the quantile-quantile plot of the delay spread versus the quantiles of the best log-normal fit. We focus on classes 4 and 9 . The first is representative of classes that show a log-normal delay spread. The second is representative of the complementary set of classes. The log-normal behavior holds true if the samples lie on the straight line. We note that the main deviation from the log-normality of class 4 is due to the samples of the lower tails (i.e., the smaller values of the delay spread).
TABLE IV

AVERAGE AND STATISTICAL COHERENCE BANDWIDTH $(\mathrm{kHz})$

\begin{tabular}{cccc}
\hline \multirow{2}{*}{ class } & Target $[23]$ & \multicolumn{2}{c}{ Simulated } \\
& $\bar{B}_{C}^{0.9}$ & $\hat{B}_{C}^{0.9}$ & $\bar{B}_{C}^{0.9}$ \\
\hline 1 & 190 & 191.433 & 187.796 \\
2 & 106 & 119.646 & 100.168 \\
3 & 160 & 167.504 & 162.718 \\
4 & 190 & 191.433 & 193.922 \\
5 & 210 & 215.363 & 212.252 \\
6 & 220 & 239.292 & 237.521 \\
7 & 370 & 382.867 & 418.282 \\
8 & 550 & 550.371 & 677.148 \\
9 & 1220 & 1268.25 & 1662.89 \\
\hline
\end{tabular}

From the analysis of experimental data (e.g., [22]), it has also been shown that the rms delay spread of measured channels is not strictly log-normally distributed, although the log-normal fit is the best one. Thus, our simulation results are consistent with previous experimental work.

As for the delay spread, we address the statistics of the average channel gain (ACG). We focus on the $\mathrm{dB}$ version of the ACG, and we define it as follows:

$$
G=10 \log _{10}\left(\frac{1}{B_{2}-B_{1}} \int_{B_{1}}^{B_{2}}|H(f)|^{2} d f\right)[\mathrm{dB}] .
$$

In Table III, we report the average value of the ACG of the nine classes (fourth column). We denote it with $\bar{G}$. The attenuation increases toward class 1 . Furthermore, except for classes 2,4 , and $8, G$ is normally distributed, as confirmed by the Lilliefors test. In Fig. 5(b), we provide the quantile-quantile plot of $G$ in decibels versus the standard normal quantiles. Again, we limit the analysis to two representative classes (i.e., class 3 and 8). Few outliers are responsible for deviation from normality of the $G$ samples of class 8 . However, the normal distribution is the best fit for the ACG of the generated channels. This result is in agreement with the experimental results in [18].

Finally, we study the coherence bandwidth. In Table IV, we report the statistical and average coherence bandwidth of the generated channels (third and fourth column, respectively), and the average coherence bandwidth of the measured channels (second column). The measured values are given in [23]. We note that the three values are very similar. Thus, the use of the average coherence bandwidth in the fitting procedure is justified.

\section{Composition Channel}

With the proposed channel generator, channels of a given class can be randomly drawn using a proper set of parameters. To generate channels that capture the overall in-home channel variability, we can randomly pick channels from all classes according to a certain occurrence probability. We refer to the resulting channel as composition channel.

To study the statistics of this composition channel, we generate 500 channels picked from different classes according to the class occurrence probability reported in [17] (i.e., $P_{o} \in$ $\left\{\begin{array}{llllllll}0.0349 & 0.1678 & 0.1818 & 0.1188 & 0.1188 & 0.1258 & 0.0979 & 0.0769\end{array}\right.$ 


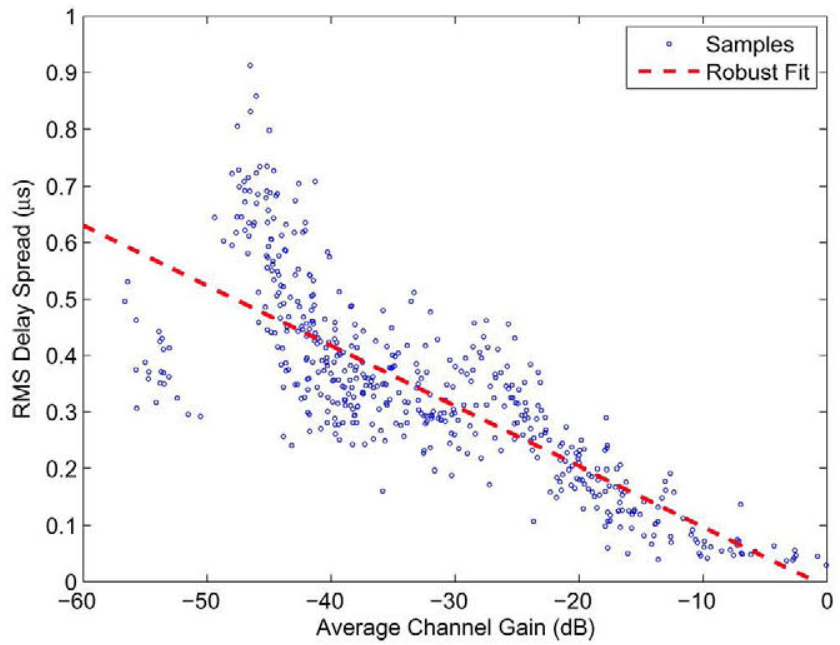

Fig. 6. Scatter plot of the rms delay spread versus the average channel gain of the composition channel. The robust fit is also shown.

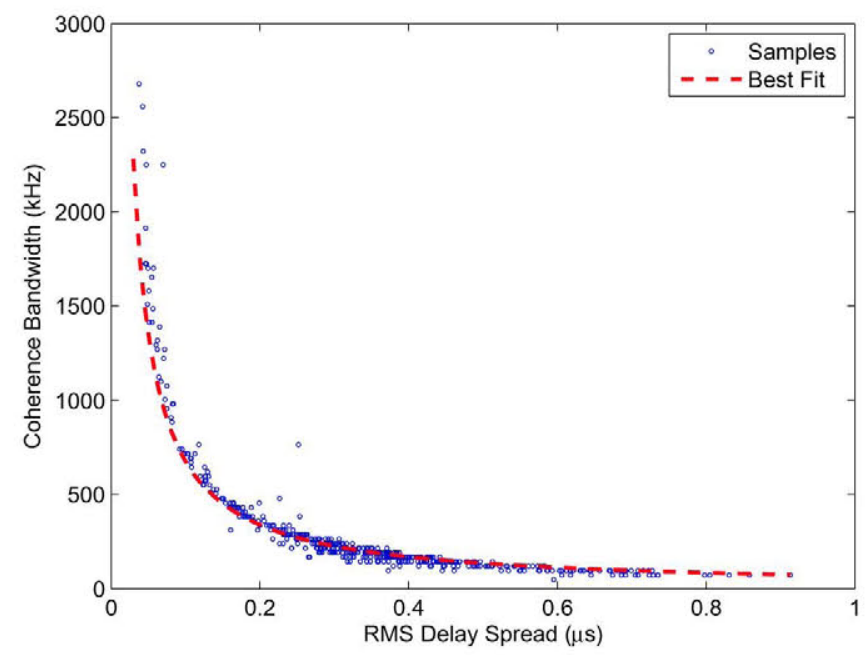

Fig. 7. Scatter plot of the coherence bandwidth versus the rms delay spread of the composition channel. The best hyperbolic fit is also shown.

$0.0769\}$ for classes 1 to 9 , respectively). The class occurence probability has been obtained from the experimental evidence in France. We focus on the ACG, rms delay spread, coherence bandwidth, and their relation. The results are summarized in Figs. 6 and 7.

In Fig. 6, we provide the relation between the ACG and the delay spread. The robust regression fit is also shown. We have found that the delay spread and the ACG are negatively related and the slope of the robust regression is $-0.011 \mu \mathrm{s} / \mathrm{dB}$. We note that this value is close to the one that has been obtained from the analysis of measured channels in [15].

Now, we focus on the coherence bandwidth. In Fig. 7, we show the coherence bandwidth as a function of the delay spread. We have found that the best fit is given by $B_{C}^{(0.9)}=0.067 \sigma_{\tau}$. In [23], a similar relation has been obtained for the measured channels, namely, $B_{C}^{(0.9)}=0.055 \sigma_{\tau}$. Hence, the close match between the experimental results and simulations validates the modeling approach.

\section{CONCLUSION}

We have addressed the random generation of PLC channel responses with statistics in agreement with that of measured channels. We have followed the top-down approach. Firstly, we have described the multipath propagation model, from which the analytical expression of the PLC channel frequency response can be derived. Then, we have introduced the variability into a restricted set of model parameters to obtain a random channel generator algorithm. We have derived the closed expression of the statistical frequency correlation function and the mean path loss profile of the generated channels. Hence, we have found the values of the model parameters that allows generating channels in good agreement with the experimental ones. To this aim, we have presented a novel fitting procedure. The procedure targets the average path loss profile and the average coherence bandwidth of the measured channels.

To test the model, we have targeted a set of measured channels whose statistics is available in the literature. The measured channels have been divided into nine channel classes according to their capacity in AWGN conditions. For each class, the average path loss profile and the average delay spread are given. We have fitted the model to all the nine channel classes, and we have provided the model parameters. Furthermore, we have studied the statistics of the generated channels, and we have found strong agreement with that from measurements. This validates the model and the fitting procedure.

Finally, we point out that although the model is intended for in-home PLC, it may be extended to other application scenarios. For instance, outdoor low-voltage PLC which will cover an important role in the Smart Grid.

\section{APPENDIX \\ DERIVATION OF THE STATISTICAL FREQUENCY CORRELATION FUNCTION}

In this section, we derive the closed-form expression of the statistical correlation function in (9). We start from (5), we model the random parameters as described in Section II-B, and we compute (8). We exploit the statistical independence of the path gains $c_{i}$ and $g_{i}$ to obtain

$$
\begin{aligned}
& \phi(f, \lambda)=|A|^{2} \sigma_{g}^{2}\left(1+b_{0}^{2} f^{K_{2}}(f+\lambda)^{K_{2}}\right) \\
& \times E\left[\sum_{i=1}^{N_{p}} e^{-j(2 \pi \lambda / \nu) \ell_{i}} e^{-\left(2 a_{0}+a_{1}\left(f^{K}+(f+\lambda)^{K}\right)\right) \ell_{i}}\right]
\end{aligned}
$$

where the expectation targets the number of paths and their lengths. We further introduce the following notation: $\alpha(f, \lambda)=$ $2 a_{0}+a_{1}\left(f^{K}+(f+\lambda)^{K}\right)$ and $\beta(\lambda)=2 \pi \lambda / \nu$. Now, the path lengths are modelled as independent and uniformly distributed random variables between 0 and $L$. Therefore, (17) turns into

$$
\begin{aligned}
\phi(f, \lambda)=|A|^{2} \sigma_{g}^{2}(1+ & \left.b_{0}^{2} f^{K_{2}}(f+\lambda)^{K_{2}}\right) \\
& \times \frac{1-e^{-(\alpha(f, \lambda)+j \beta(\lambda)) L}}{(\alpha(f, \lambda)+j \beta(\lambda)) L} E\left[N_{p}\right]
\end{aligned}
$$


Finally, we recall that the number of paths is modeled as a Poisson random variable with modified alphabet (see Section II-B). Therefore, the expectation in (18) reads

$$
E\left[N_{p}\right]=\frac{\Lambda L}{1-e^{-\Lambda L}} .
$$

Substituting (19) into (18), as well as the terms $\alpha(f, \lambda)$ and $\beta(\lambda)$, we obtain (9) (i.e., the statistical correlation function of the generated channels).

\section{REFERENCES}

[1] Home Plug Alliance. [Online]. Available: www.homeplug.org

[2] V. Oksman and S. Galli, "G.hn: The new ITU-T home networking standard," IEEE Commun. Mag., vol. 47, no. 10, pp. 138-145, Oct. 2009.

[3] S. Galli and T. C. Banwell, "A deterministic frequency-domain model for the indoor power line transfer function," IEEE J. Sel. Areas Commun., vol. 24, no. 7, pp. 1304-1316, Jul. 2006.

[4] H. Meng, Y. L. Guan, C. L. Law, P. L. So, E. Gunawan, and T. Lie, "Modeling of transfer characteristics for the broadband power line communication channel," IEEE Trans. Power Del., vol. 19, no. 3, pp. 1057-1064, Jul. 2004.

[5] J. Anatory, N. Theethayi, and R. Thottappillil, "Power-line communication channel model for interconnected networks-Part I: Two-conductor system," IEEE Trans. Power Del., vol. 24, no. 1, pp. 118-123, Jan. 2009.

[6] A. M. Tonello and T. Zheng, "Bottom-up transfer function generator for broadband PLC statistical channel modeling," in Proc. IEEE Int. Symp. Power Line Commun. App., Apr. 2009, pp. 7-12.

[7] T. Esmailian, F. R. Kschischang, and P. G. Gulak, "In-building power lines as high-speed communication channels: Channel characterization and a test channel ensemble," Int. J. Commun. Syst., vol. 16, no. 5, pp. 381-400, Jun. 2003.

[8] A. M. Tonello and F. Versolatto, "Bottom-up statistical PLC channel modeling-Part I: Random topology model and efficient transfer function computation," IEEE Trans. Power Del., vol. 26, no. 2, pp. 891-898, Apr. 2011.

[9] F. J. Cañete, J. A. Cortés, L. Díez, and J. T. Entrambasaguas, "A channel model proposal for indoor power line communications," IEEE Commun. Mag., vol. 49, no. 12, pp. 166-174, Dec. 2011.

[10] J. Anatory, N. Theethayi, and R. Thottappillil, "Power-line communication channel model for interconnected networks-Part II: Multiconductor system," IEEE Trans. Power Del., vol. 24, no. 1, pp. 124-128, Jan. 2009.

[11] F. Versolatto and A. M. Tonello, "An MTL theory approach for the simulation of MIMO power line communication channels," IEEE Trans. Power Del., vol. 26, no. 3, pp. 1710-1717, Jul. 2011.

[12] H. Phillips, "Modelling of powerline communication channels," in Proc. IEEE Int. Symp. Power Line Commun. App., Mar. 1999, pp. $14-21$.

[13] M. Zimmermann and K. Dostert, "A multipath model for the powerline channel," IEEE Trans. Commun., vol. 50, no. 4, pp. 553-559, Apr. 2002.

[14] A. M. Tonello, "Wideband impulse modulation and receiver algorithms for multiuser power line communications," EURASIP J. Adv. Signal Process., vol. 2007, pp. 1-14.

[15] S. Galli, "A novel approach to the statistical modeling of wireline channels," IEEE Trans. Commun., vol. 59, no. 5, pp. 1332-1345, May 2011.

[16] B. O'Mahony, "Field testing of high speed power line communications in North American Homes," in Proc. IEEE Int. Symp. Power Line Commun. App., Mar. 2006, pp. 155-159.

[17] M. Tlich, A. Zeddam, A. Moulin, and F. Gauthier, "Indoor power-line communications channel characterization up to $100 \mathrm{MHz}$-Part I: Oneparameter deterministic model," IEEE Trans. Power Del., vol. 23, no. 3, pp. 1392-1401, Jul. 2008.

[18] S. Galli, "A simplified model for the indoor power line channel," in Proc. IEEE Int. Symp. Power Line Commun. App, Apr. 2009, pp. 13-19.

[19] A. M. Tonello, F. Versolatto, and B. Béjar, "A top-down random generator for the in-home PLC channel," in Proc. IEEE Global Commun. Conf., Dec. 2011, pp. 1-5.

[20] D. Anastasiadou and T. Antonakopoulos, "Multipath characterization of indoor power-line networks," IEEE Trans. Power Del., vol. 20, no. 1, pp. 90-99, Jan. 2005.

[21] A. M. Tonello, S. D'Alessandro, and L. Lampe, "Adaptive pulse-shaped OFDM with application to in-home power line communications," IEEE Trans. Commun., vol. 58, no. 11, pp. 3265-3276, Nov. 2010.
[22] F. J. Cañete, J. A. Cortés, L. Díez, and J. L. G. Moreno, "On the statistical properties of indoor power line channels: Measurements and models," in Proc. IEEE Int. Symp. Power Line Commun. App., Apr. 2011, pp. 271-276.

[23] M. Tlich, A. Zeddam, A. Moulin, and F. Gauthier, "Indoor powerline communications channel characterization up to $100 \mathrm{MHz}-$ Part II: Time-frequency analysis," IEEE Trans. Power Del., vol. 23, no. 3, pp. 1402-1409, Jul. 2008.

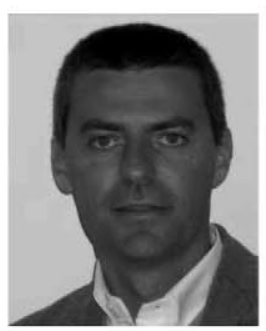

Andrea M. Tonello (M'00) received the D.Eng. degree in electronics (Hons.) and the Doctor of Research degree in electronics and telecommunications from the University of Padova, Padova, Italy.

From 1997 to 2002, he was with Bell LabsLucent Technologies, first as a member of the technical staff, then he was promoted to Technical Manager, and then to Managing Director of Bell Labs Italy. In 2003, he joined the University of Udine, Udine, Italy, where he is an Aggregate Professor. He serves as an Associate Editor for the IEEE TRANSACTIONS ON VEHICULAR TECHNOLOGY and for the IEEE TRANSACTIONS ON COMMUNICATTONS.

Dr. Tonello received several awards, including the Distinguished Visiting Fellowship from the Royal Academy of Engineering, U.K., in 2010, and the IEEE VTS Distinguished Lecturer Award 2011-2012. He was the TPC Co-Chair of IEEE ISPLC 2007, Chair of the Workshop on Power Line Communications 2009, and General Chair of IEEE ISPLC 2011. He is the Vice-Chair of the IEEE Communications Society Technical Committee on Power Line Communications.

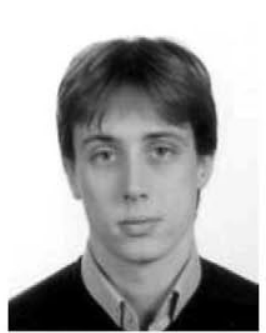

Fabio Versolatto (S'10) received the Laurea and the Laurea Specialistica degrees in electrical engineering (Hons.) from the University of Udine, Udine, Italy, in 2007 and 2009, respectively, where he is currently pursuing the Ph.D. degree.

His research interests are power-line communication channel modeling and digital communication algorithms.

Mr. Versolatto received the award for the best student paper presented at IEEE ISPLC 2010.

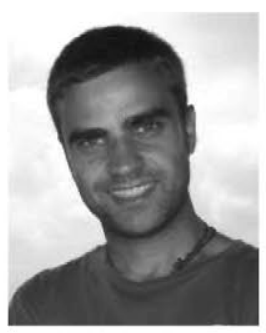

Benjamín Béjar (S'12) received the diploma degree in electrical engineering from the Universitat Politècnica de Catalunya, Spain, and the Technische Universität Darmstadt, Darmstadt, Germany, in 2006, under the framework of the double degree exchange program, and is currently pursuing the Ph.D. degree in signal processing at the Universidad Politécnica de Madrid, Madrid, Spain.

He held research appointments as a visiting Ph.D. student at the Università degli Studi di Udine, Udine, Italy, in 2009 and, recently, at the Hong Kong University of Science and Technology, Hong Kong, China, in 2011.

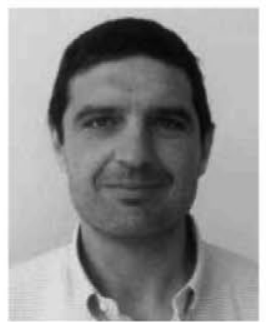

Santiago Zazo (M'12) received the Telecom Engineer and the Dr. Eng. degrees from the Universidad Politecnica de Madrid (UPM), Madrid, Spain, in 1990 and 1995 , respectively.

From 1991 to 1994 , he was with the University of Valladolid and from 1995 to 1997, he was with the University Alfonso X El Sabio, Madrid. In 1998, he joined UPM as an Associate Professor in Signal Theory and Communications. His main research activities are in the field of signal processing with applications in audio, communications, and radar. He has led many private and national Spanish projects and has participated in five European projects, leading three of them. He is leading the HF Communication Group at UPM with many activities in publications and technological transfer to civil and military applications in this field. 